\title{
Yttrium oxide passivation of porous silicon for improved photoluminescence and optoelectronic properties
}

\author{
L. Derbali ${ }^{\text {a,*, S. El whibi }}{ }^{\text {a }}$, A. Zarroug ${ }^{\text {a }}$, J. Bertomeu ${ }^{\text {b,c }}$, H. Ezzaouia ${ }^{\text {a }}$ \\ a Laboratory of Semiconductors, Nanostructures and Advanced Technology (LSNTA), Research and Technology \\ Center of Energy (CRTEn), Technopôle de Borj-Cedria, BP 95, Hammam-Lif 2050, Tunisia \\ ${ }^{\mathrm{b}}$ Department of Applied Physics, Universitat de Barcelona, 08028 Barcelona, Spain \\ ${ }^{\mathrm{c}}$ Institute of Nanoscience and Nanotechnology (IN2UB), Universitat de Barcelona, Spain \\ *Corresponding author: e-mail address: lotderb@yahoo.fr (L. Derbali). Tel.: +216 22459049.
}

\begin{abstract}
This paper reports on the effect of yttrium oxide as a novel treatment to improve the photoluminescence intensity and stability of porous silicon (PS). Yttrium oxide $\left(\mathrm{Y}_{2} \mathrm{O}_{3}\right)$ was incorporated into the PS layers by impregnation method using a saturated aqueous solution. The penetration of Yttrium into the PS microstructure was examined using the Energy Dispersive X-ray spectrometry (EDS) and the Backscattered Electron Detector (BED-C) for composition imaging and analysis. The morphology of the front surface was studied using a Field Emission Scanning Electron Microscope (FESEM). The deposited yttrium oxide onto the PS layers was thermally activated to passivate efficiently the silicon dangling bonds, and prevent the porous silicon from huge oxidation. The photoluminescence (PL) peak intensity of impregnated PS was increased noticeably compared to the as-prepared untreated PS. Unlike the as-prepared PS photoluminescence dependence with aging, the yttrium-passivated PS layers PL peak shows no shifts during aging allowing a high stability. Furthermore, we obtained a significant improvement of the effective minority carrier lifetime $\left(\tau_{e f f}\right)$ after a short anneal at $600{ }^{\circ} \mathrm{C}$, while increasing the temperature reduces noticeably the passivation properties. The improved surface passivation experienced after the thermal annealing can be ascribed to yttrium diffusion into the PS layer, with a resulting redistribution of yttrium oxide and subsequent passivation of silicon dangling bonds in the sub-interface region, this was confirmed by EDS analysis. The internal quantum efficiency (IQE) measurements were performed to study the optoelectronic properties of the processed monocrystalline silicon substrates.
\end{abstract}

Key words: Porous silicon, FESEM, Photoluminescence, passivation, Internal Quantum Efficiency (IQE), Yttrium oxide

\section{Introduction}

The visible photoluminescence (PL) from porous silicon at room temperature has attracted much attention to increase its intensity and to stabilize its emission, which enabled vast 
possibilities for technological applications [1, 2, 3]. Frequently, PS is obtained from crystalline silicon by electrochemical anodization in an aqueous hydrofluoric acid (HF) solution. In fact, the porosification of crystalline silicon allows the creation of sponge-like structures formed by pores of a few micrometers depending to the preparation method. On the other hand, porous silicon (PS) has been demonstrated to yield efficient visible light emission at room temperature over a wide range of spectra $[4,5,6]$.

Photoluminescence from PS is mostly ascribed to electron confinement in the silicon nanocrystals $[4,7]$. Furthermore, the large fraction of voids inside the porous silicon structure results in a very large specific surface area $[8,9,10]$. The main problem that many researchers are working to resolve is the instability of its native interface with metastable Siliconhydrogen termination. The latter undergoes spontaneous oxidation in ambient atmosphere and results in a degradation of the surface microstructure. Due to its poor stability in time, mainly due to the generation of nonradiative defects at the initially hydrogen-terminated nanocrystallites silicon upon the huge oxidation that proceeds during storage in air, the essential parameter to be controlled allowing a better stability of photoluminescence from PS layers is the surface state of this material. The structure of PS cannot be totally controlled [11] and PS layers can easily suffer from changes in the surface states during aging in air [12]. Many surface treatment techniques have been used to obtain stable PL or improving its intensity. One of these techniques is the passivation of PS using a thin metal oxide film deposition $[13,14,15]$. In this work, we report the beneficial effect of yttrium-passivation of porous silicon to enhance its PL stability and intensity. The effect of yttrium oxide-treated PS layers was studied and compared to other untreated PS prepared by means of the same conditions, to exhibit the effect of Yttrium on the aging behavior of PS at room temperature. The optoelectronic properties of the treated wafers were investigated. Moreover, obtained results show a noticeable increase of the Internal Quantum Efficiency (IQE) and the effective minority carrier lifetime $\tau_{\text {eff }}$, indicating the role of yttrium oxide deposited onto the PS layer, grown at the front surface, and its apparent dependence with temperature. The yttrium/PS layers can be used as an efficient silicon surface passivation layer.

\section{Experimental details}

Boron doped P-type Si (10 0) Czochralski Si (Cz-Si) wafers with 1-4 $\Omega \mathrm{cm}$ resistivity were used for the investigations reported in this work. Before starting the porosifcation process of the crystalline silicon wafers, they were cleaned using acetone then ethanol, respectively. The porous silicon layers (PS) were prepared by conventional electrochemical anodization in a 
HF-based electrolyte, the current density is $15 \mathrm{~mA} / \mathrm{cm}^{2}$ and the etching time is $10 \mathrm{~min}$, in a Teflon anodization cell with a Pt mesh as the cathode. The PS formation process was controlled using optimized parameters, particularly a constant current density that was obtained by regulating the voltage across the two electrodes connected to the potentiostat. A platinum wire were used as a counter electrode that was positioned in the electrolyte above the Si surface which is to be etched, while the metallic plate acts as the working electrode. The anodization parameters have been optimized in order to obtain PS layers with $70 \%$ porosity and around 4,3 $\mu \mathrm{m}$ thickness. After producing the porous silicon layers at the front surfaces of our Si substrates, they were rinsed in de-ionized water then immersed in a diluted aqueous HF to remove the native oxide. Subsequently, the PS were immersed in a saturated solution of yttrium oxide in diluted nitric acid $15 \% \mathrm{HNO}_{3}$, followed by a spin-on procedure in Nitrogen atmosphere at $80{ }^{\circ} \mathrm{C}$ to distribute the solution over the PS surface homogeneously. This step was repeated three times. Then, samples were slightly dried with nitrogen gas. Annealing treatment was performed first at $200^{\circ} \mathrm{C}$ for $10 \mathrm{~min}$ then at temperatures ranging between $200{ }^{\circ} \mathrm{C}$ and $700{ }^{\circ} \mathrm{C}$ for $30 \mathrm{~min}$ in nitrogen ambient, to activate the yttrium ions in the porous silicon matrix.

\section{Results and discussion}

After the electrochemical etching, the silicon substrates front surfaces were found to possess nanometer scale roughness due to the produced porous silicon layers (Fig. 1(a)) due to the formation of nanocrystalline Silicon. The thickness of the porous silicon layer was around 4,3 $\mu \mathrm{m}$. In fact, porous silicon is known to have a very large internal surface area highly reactive. The oxygen atoms react with the silicon atoms and the surface is covered with silicon dioxide even at room temperature affecting its microstructures. Micropores and metal particles are visible, as shown in fig. 1 (c, d), and seem to be distributed homogeneously onto the PS layer. After the deposition of yttrium oxide before annealing, we used two modes during the FESEM imaging to point out clearly the existence of yttrium oxide grains into the porous silicon layer: (c) is the normal mode (LED), while (d) was taken using the Backscattered Electron Detector (BED-C) mode for composition imaging and analysis. Deposited yttrium oxide is clearly shown in fig. 1 (d) apprearing in light gray and randomly distributed onto the PS layer, marked by some yellow arrows to distinguish between the porous silicon and the incorporated metal oxide. Fig. 1 (a) shows the surface morphology of the untreated PS layer. The cross-sectional FESEM view, show clearly the silicon matrix and its surface morphology. Further analysis was conducted to confirm with the Energy Dispersive Spectroscopy (EDS) 
the presence of yttrium in the PS Matrix. Fig. 2 exhibits the EDS analysis spectra of the asprepared PS layer confirming also the presence of the incorporated yttrium oxide into the porous silicon layers, before annealing.

Fig. 3 shows the variation of the Skewness $\left(\mathrm{S}_{\mathrm{sk}}\right)$ and the average maximum profile valleys depth, deduced from AFM analy ${ }^{2}$ sis. They are important in giving a better understanding of the surface. Skewness $\left(\mathrm{S}_{\mathrm{sk}}\right)$ is a measure of the asymmetry of the distribution of heights about its mean line, when it is symmetrical its value is zero. Negative values of $\mathrm{S}_{\mathrm{sk}}$ indicate that the surface is more planar and valleys are predominant. In fact, if the surface has more peaks than valleys and the height distribution is asymmetrical the Skewness is positive $[16,17]$.

Accordingly, as shown in fig. 3 , the obtained values of $S_{\text {sk }}$ prove that the surface contains a high concentration of pores that tend to decrease after annealing the immersed samples. This is due to the pore filling with yttrium oxide that took place after increasing the annealing temperature to $500{ }^{\circ} \mathrm{C}$, then $\mathrm{S}_{\mathrm{sk}}$ decreases obviously. The decrease of the skewness for higher temperatures can be related to the diffusion of the incorporated yttrium oxide deep into the pores due to the thermal treatment effect $[13,14]$.

Due to the importance of the textured surface analysis to study the morphology of the prepared samples, we plotted the variation of the average maximum profile valley depth $\left(\mathrm{R}_{\mathrm{vm}}\right)$ in fig. 3. The latter is defined as the measure of the deepest pore (described as valley) depths in the yttrium-treated porous silicon, calculated over the surface $[16,18,19]$. It concerns the deepest valleys across the surface profile analyzed from the mean line. $R_{v m}$ is a useful parameter to study the morphological changes in the post-annealed yttrium/PS layers. According to fig. 3, $\mathrm{R}_{\mathrm{vm}}$ variations indicate an apparent dependence of the pores (described as valleys) dept with annealing temperature due to the induced agglomerations and pore filling that looks in accordance with the discussed variations of the skewness $\left(\mathrm{S}_{\mathrm{sk}}\right)$.

Fig. 4 shows the photoluminescence dependence of porous silicon with the subjected yttrium passivation post-annealed at different temperature. The PL intensity increases first and then decreases drastically as the temperature exceeds $600^{\circ} \mathrm{C}$. It can be seen that the highest improvement in the PS photoluminescence was obtained after the passivation of PS with yttrium oxide thin film after post-annealing at $600^{\circ} \mathrm{C}$. The PL intensity of PS is increased significantly compared to that of the uncoated PS. 
Metal-passivation on the surface of porous silicon crystallites has been reported before to have a beneficial effect $[13,14,20]$. In contrast, some passivation methods, such as sputtering for example, of certain metals does not easily result in the bonding of Si dangling bonds with metal ions, and the formed metal layer accelerates the diffusion of excited carriers for nonradiative decay in nearby defects rather than preventing. It is the purpose of the present study to use yttrium oxide passivation, thermally activated, to passivate the silicon dangling bonds, and form an yttrium oxide surface layer on the Si nanocrystallites to protect the PS layer from intense oxidation. For that reason, the silicon dangling bonds are known to have a detrimental effect on the porous silicon PL properties since they are acting as nonradiative centers that decrease obviously the PL intensity. The PL intensity dependence with temperature is depicted in fig. 5.

Fig 6 shows FTIR spectra of the treated PS with yttrium oxide and post-annealed at $600^{\circ} \mathrm{C}$. Yttrium oxide was clearly identified via its characteristic bonds, indicating the crucial role of yttrium in the passivation process of the nanocrystallites in the PS layer. The formation of Y O-Si bonds have been reported and proved in previous studies [21]. The characteristic vibration bands of the Y-O bond (metal-oxygen) were observed at 562, $455[22,23]$ and 506 $\mathrm{cm}^{-1}[24,25]$. These bands have already been reported for $\mathrm{Y}_{2} \mathrm{O}_{3}$ thin films obtained by other techniques [23]. Specific bands for Y-O-Si, O-Si-O and Y-O bonds are observed below 594 $\mathrm{cm}^{-1}$ [21]. Besides, absorbance bands located at around 810, and $1080 \mathrm{~cm}^{-1}$, which were related to the asymmetric bending, and stretching modes, respectively, of Si-O-Si bonding $[21]$.

Fig. 7 depicts the EDS analysis performed with careful characterization on the cross-section of the treated PS layer with yttrium at the optimum temperature $\left(600^{\circ} \mathrm{C}\right)$. The results point out an obvious increase of the atomic percentage of yttrium, from the top to the bottom of the layer. The latter confirms that yttrium was deeply incorporated into the porous silicon microstructure. It is worth noting that FESEM observations of the prepared porous silicon and Yttrium /PS revealed a thickness around $4.3 \mu \mathrm{m}$. We can infer that yttrium diffusion into the porous silicon microstructure and at the sub-surfacique region of the silicon substrate, as confirmed with the EDS analysis of fig. 7, has a crucial effect on the obtained improvements of the PS optoelectronic properties. 
The effect of aging on both untreated PS and yttrium-PS treated layers was investigated. After storing the passivated PS with yttrium oxide in ambient air at room temperature, there is no PL blueshift observed, as shown in fig. 8 (a). For comparison, untreated PS was also prepared by the same technique and same conditions. As shown in fig. 8 (b), not only blueshift in PL peak positions was observed during storing, but also a slight decrease of intensity. The modified silicon nanocrystallites, after the incorporation of yttrium oxide, consist of three regions: the Si core, interface, and the outer Y layer. The surface layer is transformed to a contaminated native oxide, during exposure to air, while the PL undergoes significant degradation. In fact, in the interface between the inner core and outer silicon oxide layer the oxidation process producing dangling bonds and other nonradiative centers. This may explain the reduced intensity and the resulting blueshift that occurs during storing the as-prepared untreated PS in air.

Our results clearly show that the yttrium-passivated PS surface structure has a distinct advantage. First, the interface is well terminated by yttrium ions, forming a perfect interface structure rather than that in normal oxidized PS. Second, the outer yttrium oxide layer can prevent a possible large diffusion of oxygen into the crystalline silicon core, which can decrease the nanocrystallites size and also leads to the creation of silicon dangling bonds. This is the reason why in yttrium passivated PS the PL was enhanced significantly and stabilized without remarquable blueshift during aging. Accordingly, the improved PL intensity from yttrium-passivated PS layer of fig. 4 is due mainly to surface dangling bonds being further terminated by yttrium ions. The schematic illustration in fig. 9 show the effect of postannealing on the penetration and diffusion of yttrium oxide into the pores, also, the silicon nanocrystallites is suggested to be covered with the yttrium oxide, as shown in fig. 9(b), which explains the efficiency of its passivation effect of the PS layer. The thermal activation stimulated the diffusion of impurities towards the outer surface of the silicon nanocrystallites. The outer yttrium oxide layer on the surface can prevent ambient oxygen from migrating into the Si core of the PS microstructure and consequently its size was undiminished, and therefore the peak position is found stable. These results suggest that the photoluminescence from aged yttrium-PS might be ascribed to the combined effects of quantum confinement in silicon nanocrystallites and radiative recombination occurring through different types of luminescence centers such as complexes of silicon with impurities or point defects and impurities in which the incorporated yttrium ions can play a crucial role, without any peak shift dependence according to the proposed model of Qin and Jia [26, 27]. 
The total reflectivity was measured by means of Perkin-Elmer Lambda spectrophotometer equipped with an integrating sphere. Fig. 10 shows the reflection spectra for the as-prepared untreated PS, the as-deposited yttrium oxide onto the PS layer and the post-annealed yttriumPS at the optimum temperature $600^{\circ} \mathrm{C}$. It can be seen that the reflectivity of the PS layer was reduced to its lower values after annealing at $600{ }^{\circ} \mathrm{C}$. The increase of temperature leads usually to a variation of the morphology, which notably reduces the reflectivity. The reduced reflectivity can be partially explained by an increased roughness after the incorporation of yttrium oxide into the PS layer. It is well known that surface morphologies with high roughness, allowing a high scattering and therefore a resulting low reflectivity. This behavior is mainly attributed to the high internal multiple reflections into the porous structure, as illustrated in fig. 9 (a), known to trap efficiently the incident light.

The combined effect of the post-annealed yttrium/PS at various temperatures acts as a passivating layer of the crystalline silicon surface and also an efficient antireflection layer increasing consequently the absorption of the incident light at the front surface. The latter was investigated by means of the Internal Quantum Efficiency (IQE) and the effective minority carrier lifetime measurements. Results show clearly the effect of yttrium oxide on the passivation process of the PS layers and its dependence with temperature, compared with the as-prepared untreated PS subjected to the same thermal treatment in the same conditions. The effective minority carrier lifetime shows a minor improvement in the as-prepared untreated PS layers subjected to the same thermal annealing, as shown in fig. 11, with maximum values not exceeding $8 \mu \mathrm{s}$. Concerning The PS/Yttrium treatment post-annealed at various temperatures, measured $\tau_{\text {eff }}$ reach its highest values $37 \mu$ s after annealing PS/Yttrium at $600^{\circ} \mathrm{C}$, showing a good agreement the discussed results in the PL study. Thus, carrier lifetime was significantly improved by the proposed Yttrium-PS treatment, in view of the fact that recombination activities have been reduced significantly allowing the obtained increase of the effective minority carrier lifetime. Lee and Glunz [28] showed that improve carrier lifetime also improved the solar cell efficiency. This was reported in many previous works since improving the electrical properties of crystalline silicon is of great importance for photovoltaic applications [29, 30].

In point of fact, the front surface reflectance can be reduced by means of texturization, but due to an increase in the number of surface defect states the recombination activities will rise. In view of that, the proposed yttrium-passivation treatment of PS layer, grown at the front surface, aims to reduce the recombination activities and to improve the surface quality. As 
discussed, obtained results prove the beneficial effect of the deposited yttrium oxide which passivated the surface defect states efficiently. To investigate furthermore the effect of yttrium-PS treatment we measured the internal quantum efficiency (IQE) after each thermal annealing, presented in fig. 12. An internal quantum efficiency spectrum can be defined as an involvement of the known three parts of a pn junction, namely the emitter, space charge and base [31]. Wavelengths less than $600 \mathrm{~nm}$ can penetrate only up to a few micrometers into the silicon substrate, consequently, a large number of charge carriers in the emitter and close to the pn junction regions will be generated. The recombination activities at the front surface in silicon has a significant effect on the generated carriers collection probability in the short wavelength range, since it is well known that recombination has a detrimental effect on the electrical properties of silicon solar cells. According to the IQE spectra, in fig. 12 it is apparent that the major difference between the annealed samples appears in the wavelength region $400-650 \mathrm{~nm}$, confirming that $600^{\circ} \mathrm{C}$ is the optimal annealing temperature and found to be consistent with our discussed results. Improvements are also significant between 650 and around $900 \mathrm{~nm}$, indicating an obvious improvement of the electrical properties in the bulk. The effect of yttrium-PS passivation through a thermal annealing enable a non negligible thermal agitation of impurities to facilitate its migration near the surface were they will be trapped into the porous silicon layers and at the interface, which increases the recombination activities, as illustrated in fig. 9(b). The latter can explain the PL drop after annealing at $700^{\circ} \mathrm{C}$ and seems to be in good agreement with the variations of the effective minority carrier lifetime of fig. 11 .

\section{Conclusion}

In order to gain insight into the porous silicon properties after the yttrium-PS treatment, we studied the effects of thermal treatment and aging in air ambient. The deposited yttrium oxide exhibits a crucial role on the stabilization of PL during aging and improves its intensity after a post-annealing at an optimum temperature, which was found $600^{\circ} \mathrm{C}$. Obtained results prove the beneficial effect of yttrium-PS passivation on the PL properties of porous silicon and its effect on the improvement of the electrical properties in the corresponding crystalline silicon substrate, regarding essentially the important improvement of the effective minority carrier lifetime that reached $37 \mu \mathrm{sec}$ after annealing at $600^{\circ} \mathrm{C}$, and the IQE measurements. Hence, yttrium oxide incorporation into porous silicon is a promising process for porous silicon to realize enhanced silicon-based optoelectronic devices. 
Acknowledgements: This work was supported by the Ministry of Higher Education and Scientific Research of Tunisia, and the Spanish Ministerio de Economía, Industria y Competitividad and the European Regional Development Fund through the project ENE201678933-C4-2-R. We would like to thank Dr. J. Andreu and J. Miguel (University of Barcelone) for the support and the fruitful discussions. Also, we are grateful to Á. Lorenzo for the helpful assistance and support during some characterizations.

\section{References}

[1] D. Kovalev, H. Heckler, G. Polisski, and F. Koch, Optical properties of Si nanocrystals, Phys. Status Solidi B 215 (1999) 871

[2] B. Gelloz, A. Loni, L. Canham, and N. Koshida, Luminescence of mesoporous silicon powders treated by high-pressure water vapor annealing, Nanoscale Res. Lett. 7, 382 (2012).

[3] S. Takeoka, M. Fujii, and S. Hayashi, Size dependent photoluminescence from surfaceoxidized Si nanocrystals in a weak confinement regime, Phys. Rev. B 62, 16820 (2000).

[4] O. Bisi, S. Ossicini, L. Pavesi, Porous silicon : a quantum sponge structure for silicon based optoelectronics, Surf. Sci. Rep. 38 (2000) 1.

[5] A.G. Cullis, L.T. Canham, P. D. J. Calcott, The structural and luminescence properties of porous silicon, J. Appl. Phys. 82 (1997) 909.

[6] L.T. Canham, in: L.T. Canham (Ed.), Properties of Porous Silicon, INSPEC, London, 1997, p. 249.

[7] P.M. Fauchet, Photoluminescence and electroluminescence from porous silicon, J. Lumin. 70 (1996) 294.

[8] C. Vinegoni, M. Cazzanelli, L. Pavesi, in: H.S. Nalwa (Ed.), Silicon-Based Materials and Devices, Properties and Devices, vol. 2, Academic Press, 2001, p. 123.

[9] L.T. Canham, in: L.T. Canham (Ed.), Properties of Porous Silicon, INSPEC, London, 1997, p. 83.

[10] R. Herino, in: L.T. Canham (Ed.), Properties of Porous Silicon, INSPEC, London, 1997, p. 89.

[11] R. L. Smith and S. D. Collins, Porous silicon formation mechanisms, J. Appl. Phys. 71, R1 (1992). 
[12] T. Ito and A. Hiraki, Aging phenomena of light emitting porous silicon, J. Lumin. 57, 331 (1993).

[13] L. Derbali, H. Ezzaouia, Electrical properties improvement of multicrystalline silicon solar cells using a combination of porous silicon and vanadium oxide treatment, Appl. Surf. Sci, 271 (2013) 234-239.

[14] R. Riahi, L. Derbali, B. Ouertani, H. Ezzaouia. Temperature dependence of Nickel Oxide effect on the optoelectronic properties of porous silicon, Appl. Surf. Sci, 404 (2017) 34-39.

[15] Ahmed Zarroug, Ikbel Haddadi, Lotfi Derbali, Hatem Ezzaouia. LiBr treated porous silicon used for efficient surface passivation of crystalline silicon solar cells, Superlattices and Microstruct. 80 (2015) 181-187.

[16] Anai A. Valencia-Lazcano, Teresa Alonso-Rasgado, Ardeshir Bayat. "Characterisation of breast implant surfaces and correlation with fibroblast adhesion", J. Mech. Behav. Biomed. Mater. 21 ( 2013 ) $133-148$.

[17] Hansson S. Surface roughness parameters as predictors of anchorage strength in bone: a critical analysis. J Biomech Eng 2000;33:1297.

[18] R.R.L. De Oliveira, D.A.C. Albuquerque, T.G.S. Cruz, F.M. Yamaji and F.L. Leite. "Measurement of the Nanoscale Roughness by Atomic Force Microscopy: Basic Principles and Applications", Atomic Force Microscopy - Imaging, Measuring and Manipulating Surfaces at the Atomic Scale, Dr. Victor Bellitto (Ed.), 2012, InTech, DOI: 10.5772/37583. Available from: https://www.intechopen.com/books

[19] P Vitanov, A Harizanova, T Ivanova and H Dikov. « Low-temperature deposition of ultrathin $\mathrm{SiO} 2$ films on Si substrates », Journal of Physics: Conference Series 514 (2014) 012010 .

[20] Hong, C., Kim, H., Kim, H.W. et al. Enhancement of the Photoluminescence of Porous Silicon by Sputter Deposition of Semitransparent Metal Films, Met. Mater. Int. 16 (2010) 311-315.

[21] J. J. Chambers and G. N. Parsons, Physical and electrical characterization of ultrathin yttrium silicate insulators on silicon, J. Appl. Phys. 90 (2001) 918.

[22] Hock Jin Quah, Kuan Yew Cheong, Effects of post-deposition annealing ambient on $\mathrm{Y}_{2} \mathrm{O}_{3}$ gate deposited on silicon by RF magnetron sputtering, J. Alloy. Compd. 529 (2012) 73 83.

[23] H. Guo, W. Zhang, L. Lou, A. Brioude, J. Mugnier, Structure and optical properties of rare earth doped $\mathrm{Y}_{2} \mathrm{O}_{3}$ waveguide films derived by sol-gel process, Thin Solid Films 458 (2004) 274-280. 
[24] S. Xiaoyi, Z. Yuchun, Preparation and optical properties of $\mathrm{Y}_{2} \mathrm{O}_{3} / \mathrm{SiO}_{2}$ powder, Rare Met. 30 (2011) 33.

[25] R. Ahlawat and P. Aghamkar, Influence of annealing temperature on $\mathrm{Y}_{2} \mathrm{O}_{3}: \mathrm{SiO}_{2}$ Nanocomposite prepared by Sol-Gel process, Acta Physica Plonica A 126 (2014) 736.

[26] G. G. Qin and Y. Q. Jia, Mechanism of the visible luminescence in porous silicon, Solid State Commun. 86, 559 (1993).

[27] Yoshio Fukuda, Kazuo Furuya, Nobuhiro Ishikawa, and Tetsuya Saito, Aging behavior of photoluminescence in porous silicon, Journal of Applied Physics 82, 5718 (1997)

[28] J. Y. Lee and S. W. Glunz, "Investigation of various surface passivation schemes for silicon solar cells “, Solar Energy Materials and Solar Cells, vol. 90, no. 1, pp. 82-92, 2006.

[29] L. Derbali, A. Zarroug, H. Ezzaouia. Minority carrier lifetime and efficiency improvement of multicrystalline silicon solar cells by two-step process. Renewable Energy 77 (2015) 331-337.

[30] Ahmed Zarroug, Lotfi Derbali, Hatem Ezzaouia. The impact of thermal treatment on gettering efficiency in silicon solar cell. Materials Science in Semiconductor Processing 30 (2015) 451-455.

[31] S. M. Sze, Physics of Semiconductor Devices, 2nd ed, Wiley, New York, 1981. 

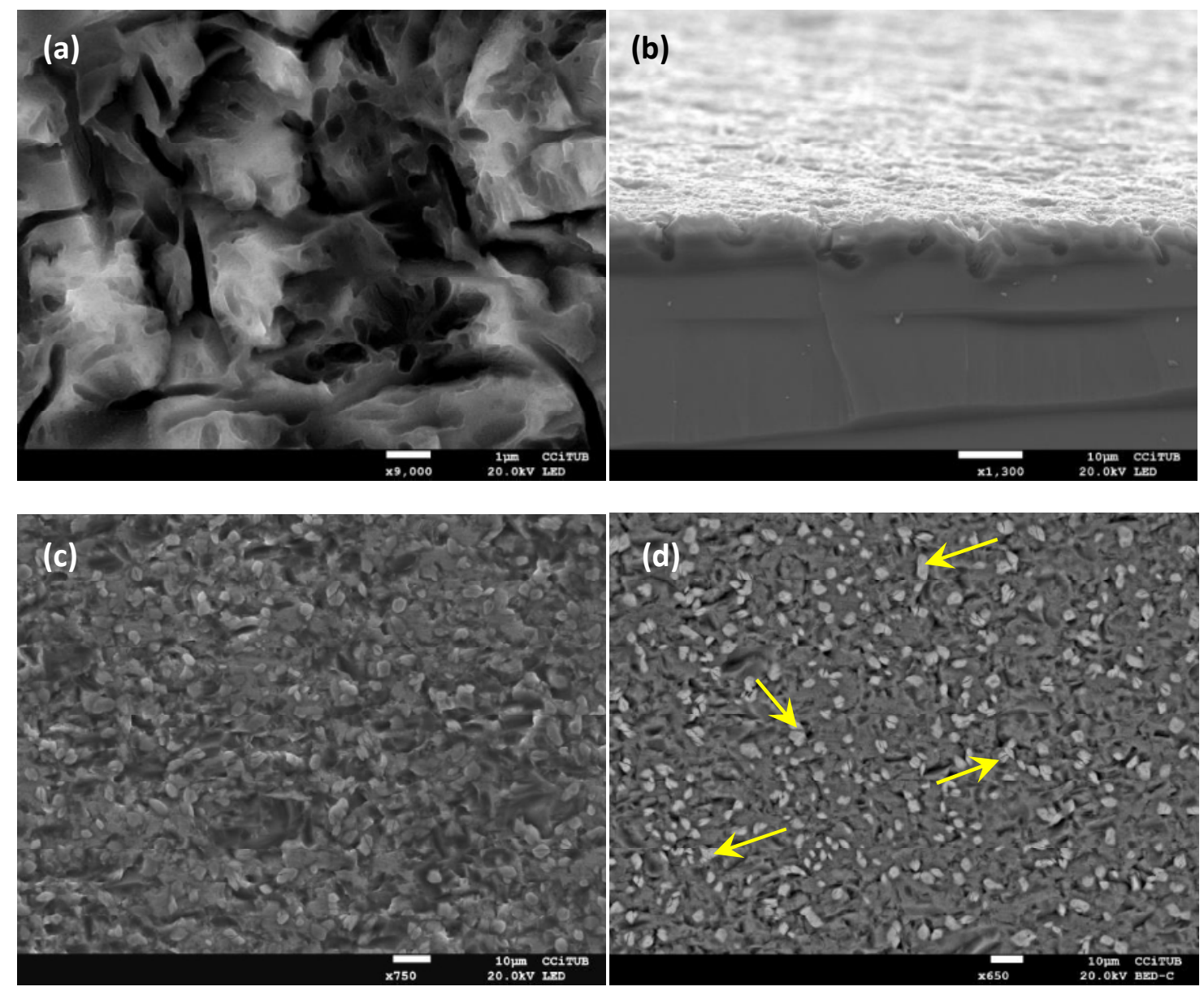

Fig. 1. SEM micrograph of the PS structure (a), cross-sectional view (b). (c) and (d) are the same sample with different imaging mode: The lower electron detector (LED) and the Backscattered Electron Detector

(BED-C) for composition imaging and analysis, respectively. (d) shows the deposited yttrim oxide nanocrystallites appearing in light gray randomly distributed onto the PS layer (marked using some yellow narrows). 


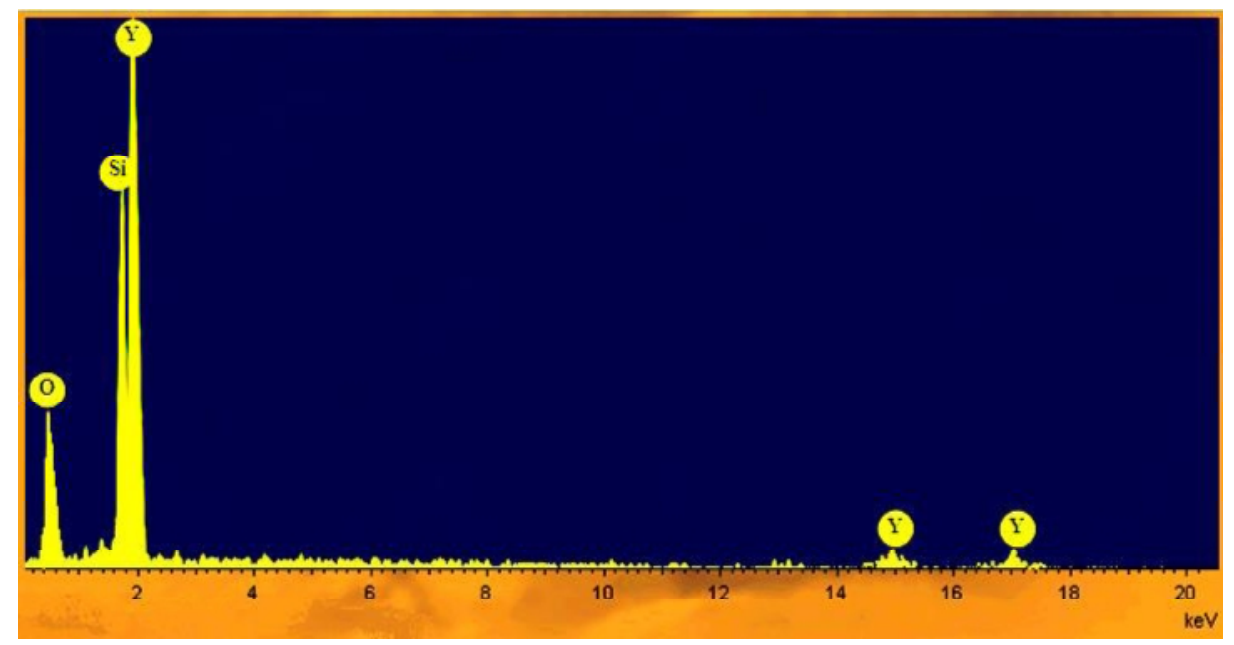

Fig. 2. Energy dispersive spectroscopy (EDS) analysis of the as-deposited yttrium oxide onto the PS layers. 


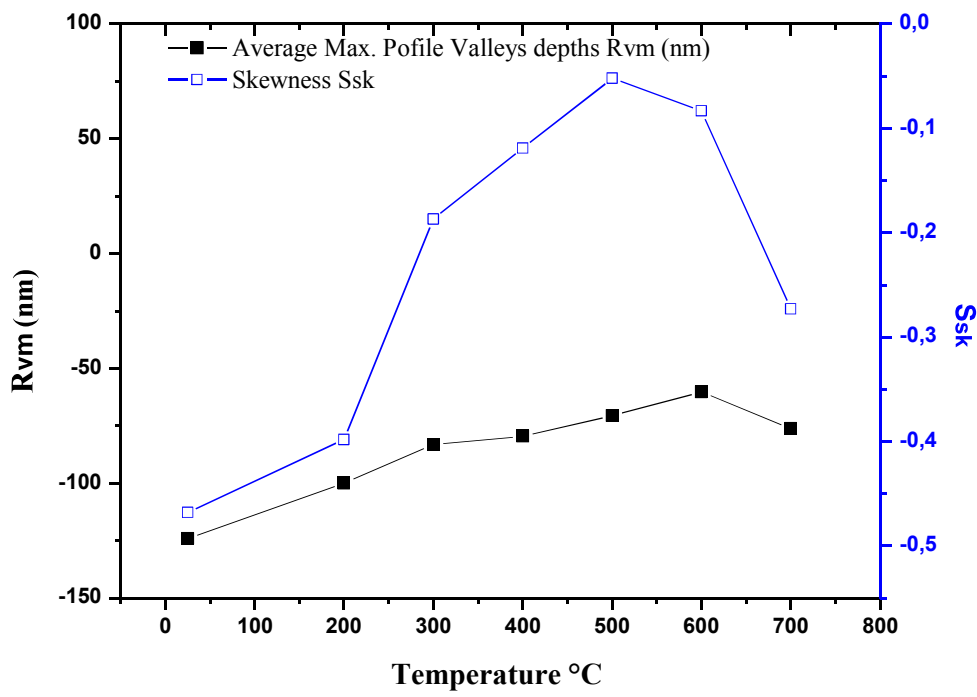

Fig. 3. Average roughness parameters of the treated porous silicon layers, deduced from AFM surface analysis: Pores depth variations and morphology profile. 


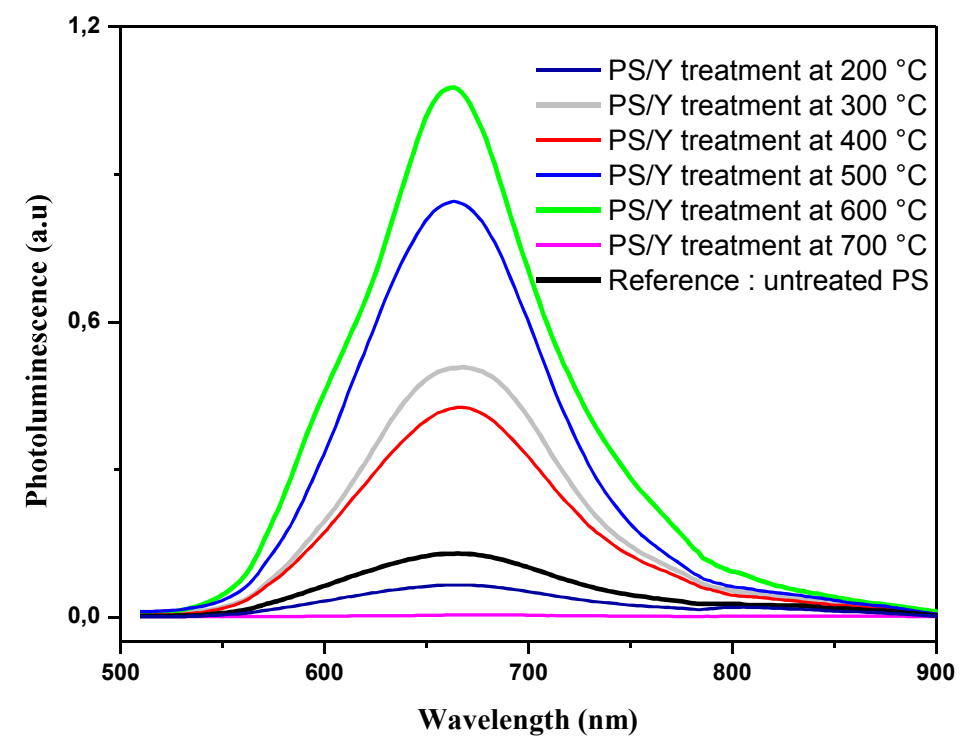

Fig. 4 PL spectra of the Yttrium-PS passivated layers annealed at different temperatures. 


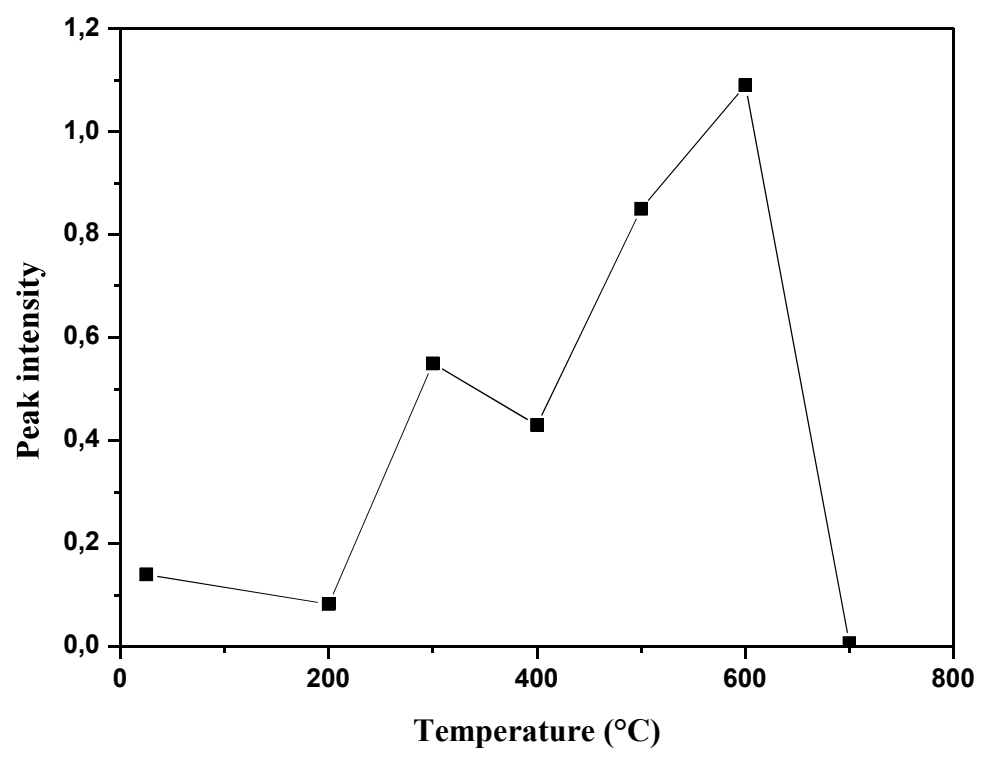

Fig. 5. Photoluminescence peak intensity dependence with thermal annealing after the yttrium-PS passivation. 


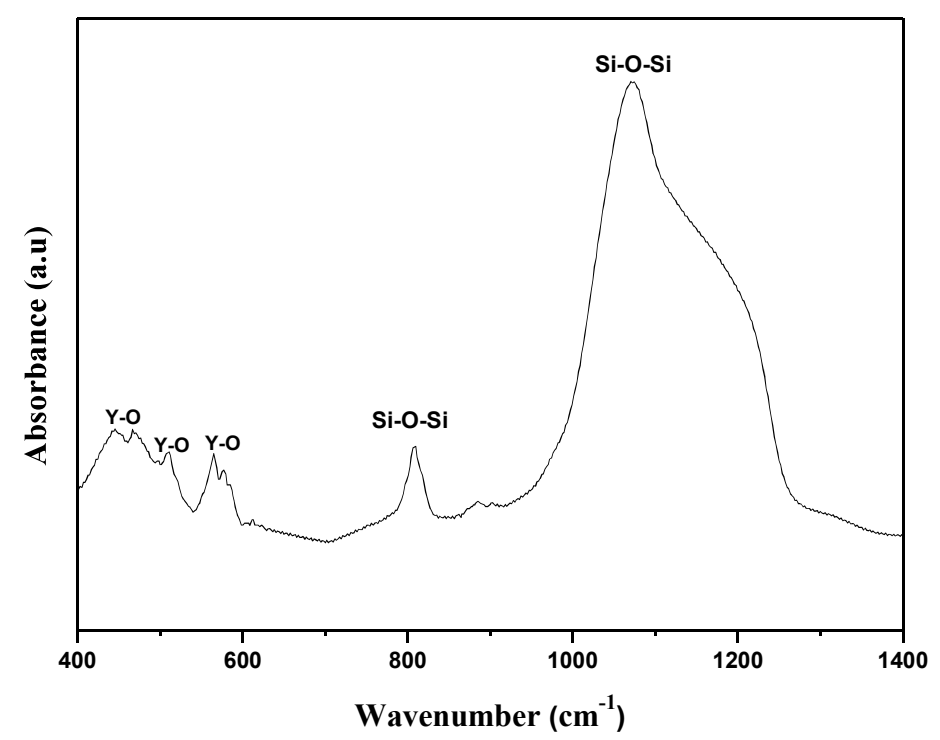

Fig. 6. FTIR spectra of the treated Y/PS layer at the optimum annealing temperature $600^{\circ} \mathrm{C}$. 


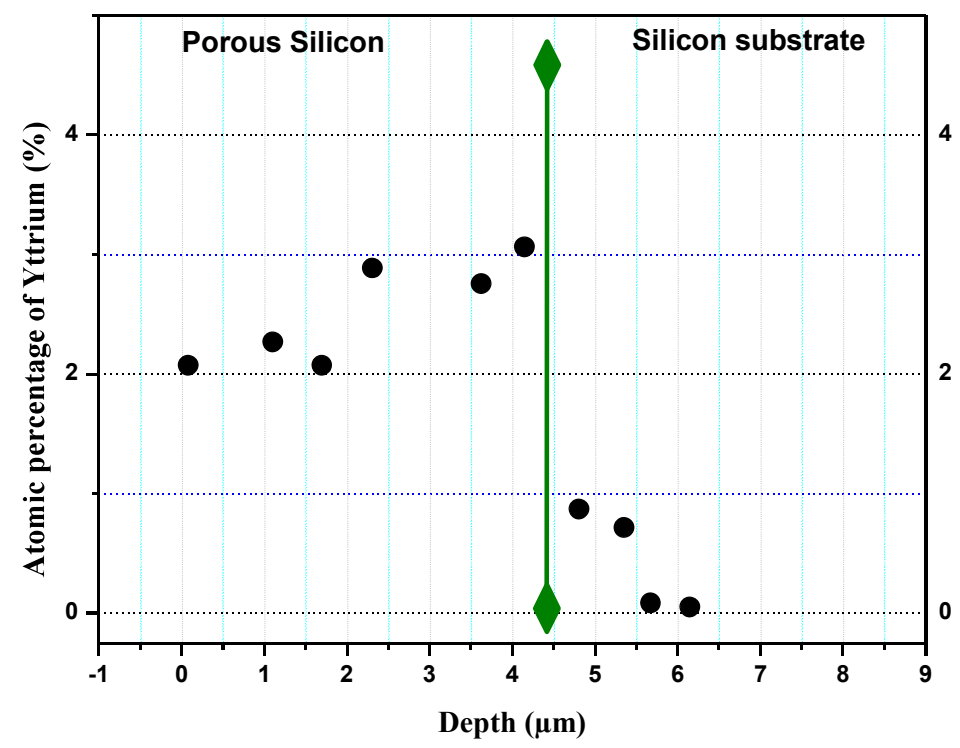

Fig. 7. Atomic percentage of yttrium obtained from a cross-section analysis, in different regions after annealing at the optimum temperature $600^{\circ} \mathrm{C}$, using EDS analyses. 

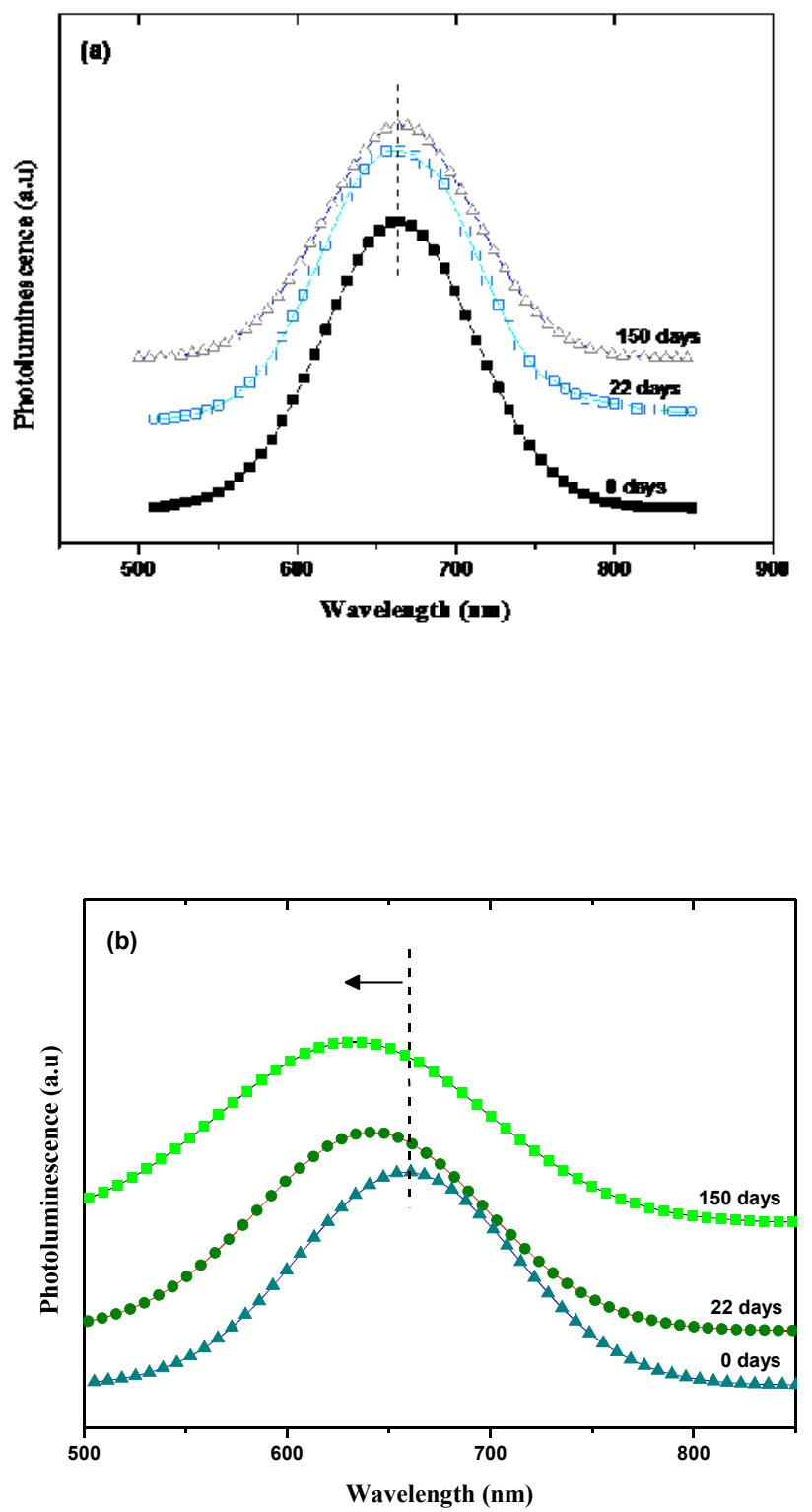

Fig. 8. Photoluminescence spectra of PS/Y annealed at $600^{\circ} \mathrm{C}$ (a), and the as-prepared untreated PS (b), after aging for 22 and 150 days. 
(a)

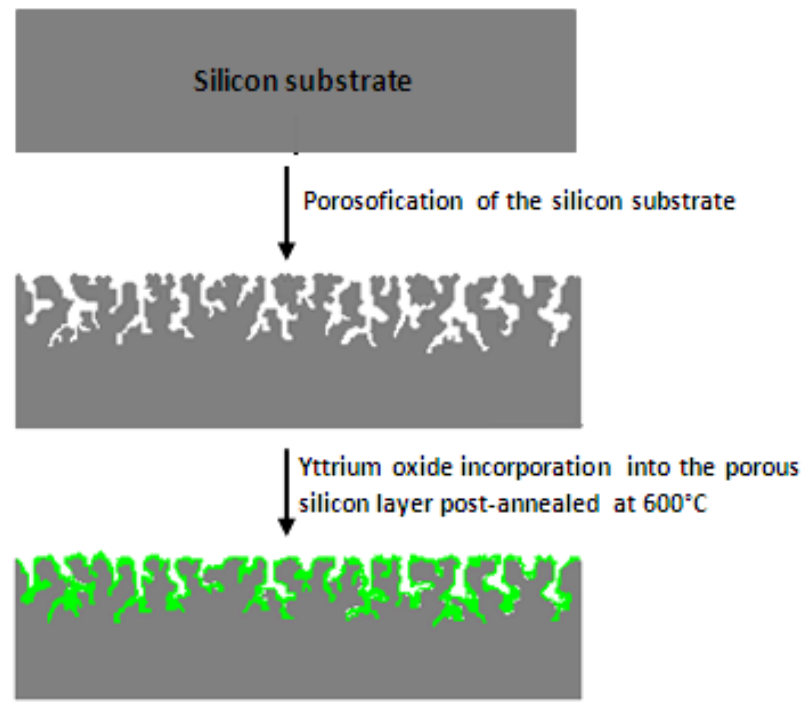

(b)

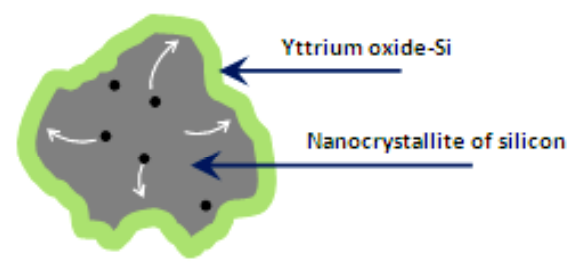

Fig. 9. Schematic illustration of the obtained formed porous silicon (a) and the effect of the thermal annealing on the migration of some impurities toward the outer surface of nanocrystallites allowing a further reduction of the recombination activities in the PS layer (b). 


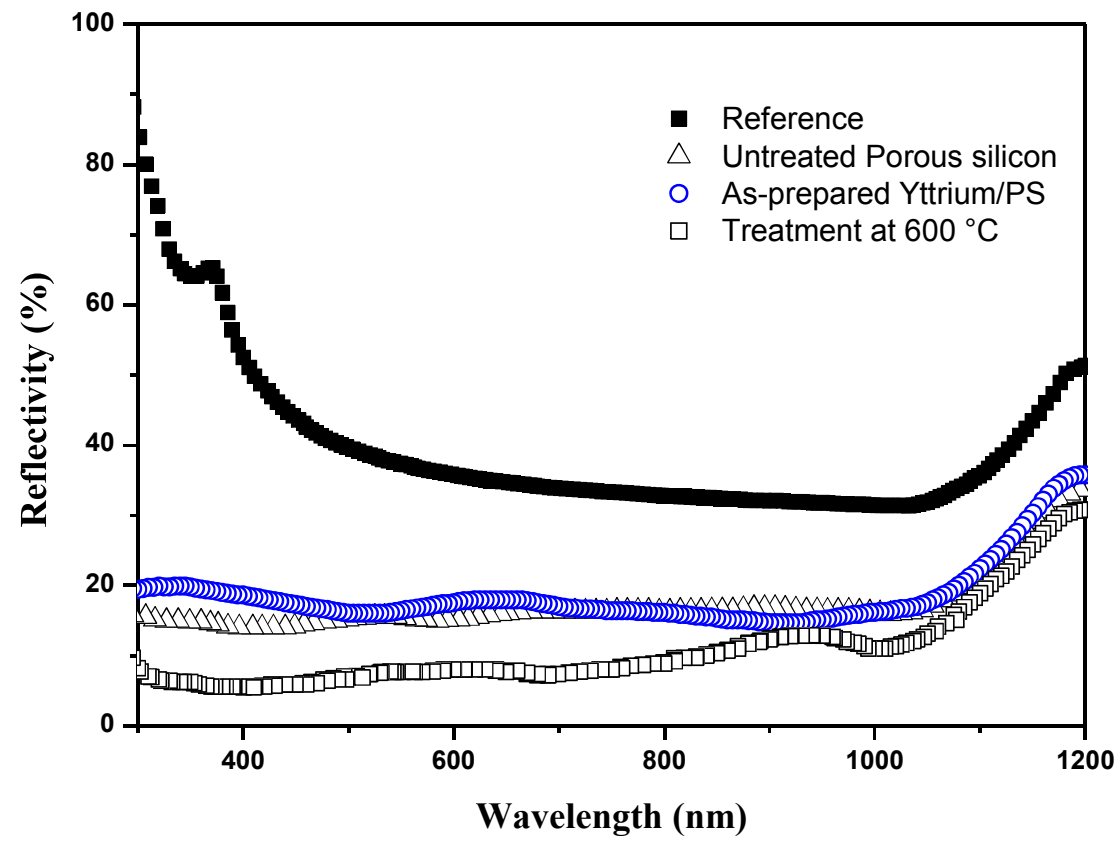

Fig. 10. Total reflectivity spectra showing the difference between an as-prepared untreated PS layer, the as-deposited yttrium oxide onto PS and the post annealed yttrium-PS at the optimum annealing temperature $\left(600^{\circ} \mathrm{C}\right)$. 


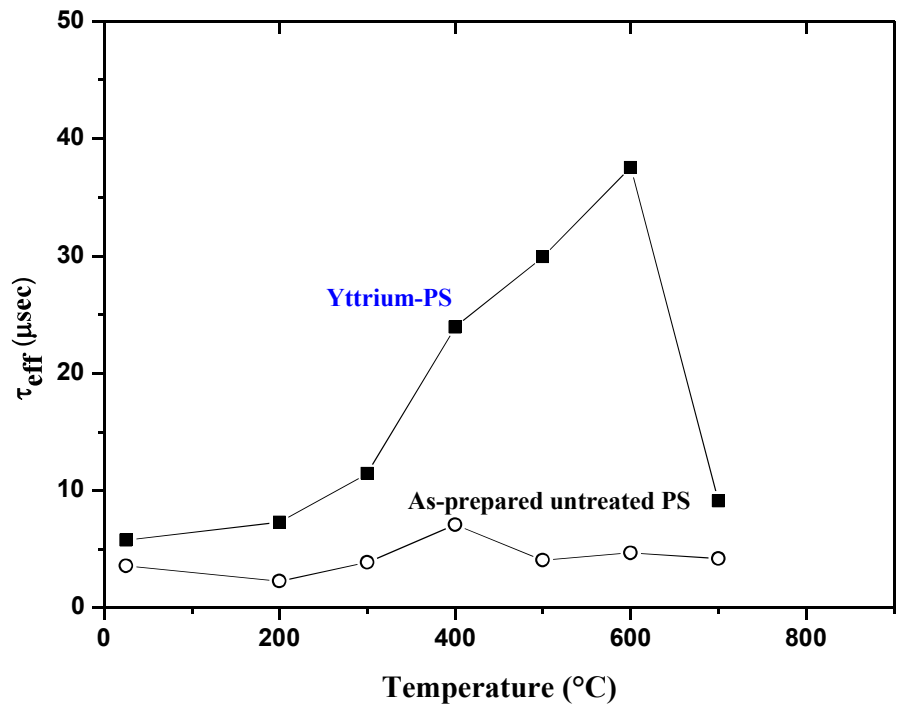

Fig. 11. Temperature dependence of the effective minority carrier lifetime $\tau_{\text {eff }}$ in the yttrium-PS passivated layer compared to an as-prepared untreated PS layer subjected to the same thermal treatment. 


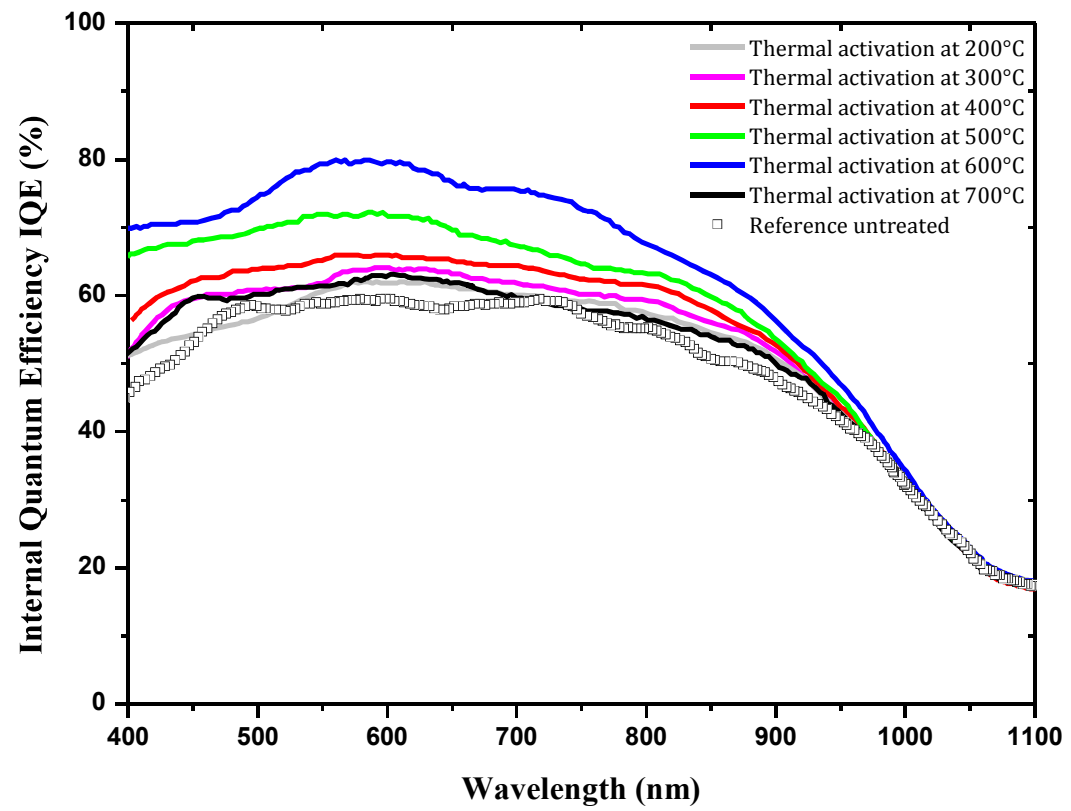

Fig. 12. Internal quantum efficiency (IQE) variations of the treated PS layers with yttrium oxide compared to a reference untreated sample. 